\title{
The Challenges of Volunteering during the COVID-19 Pandemic
}

\author{
Aleksandra Luksyte \\ Patrick D. Dunlop \\ Djurre Holtrop \\ Marylène Gagné \\ Darja Kragt \\ Hawa Muhammad Farid
}

The University of Western Australia, Perth, Western Australia and Curtin University, Perth, Western Australia

Email: alex.luksyte@uwa.edu.au

This is the prepublication version of the following manuscript:

Luksyte, A., Dunlop, P. D., Holtrop, D., Gagné, M., Kragt, D., \& Muhammad Farid, H. (2021). The challenges of volunteering during the COVID-19 pandemic. Industrial and Organizational Psychology, $14,286-289$.

C2021. This paper is not the copy of record and may not exactly replicate the authoritative document published in Industrial and Organizational Psychology 
Although Rudolph et al. (2020) outlined how the current pandemic will affect the work and life of employees, they have overlooked the potential disruption this pandemic will have on volunteers - a sizable segment of any developed economy (Volunteers, 2018) - and their volunteering experiences. Here, we define volunteering as time willingly and freely given to benefit another person, group, or cause (Wilson, 2000). We observed that volunteering has been disrupted substantially by the pandemic in our research with State Emergency Services (SES) in Western Australia, in which 96\% of emergency service personnel constitute of volunteers (Services, 2019). The current pandemic has already affected the volunteering experiences of this essential segment of the Australian economy. The pandemic restrictions have disrupted the availability of emergency services volunteers to participate in face-to-face training activities and combat impending natural hazards such as bushfires, floods, and cyclones. For example, in times of disasters, additional emergency services volunteers usually come from interstate and overseas, yet, such help is difficult to source, due to the pandemic imposed travel restrictions. Further, Australian emergency services should account for the possibility of hundreds of volunteers working on the frontlines needing to be isolated if they become infected during the pandemic. In addition to these economic disruptions that the pandemic will likely cause for emergency services and other organizations employing volunteers, we argue that there are likely to be psychological disruptions and challenges. The challenges stem partly from the need to comply with the pandemic imposed restrictions, and partly from the unique features of volunteering work.

Rudolph et al. (2020) have discussed 10 areas, which will be mostly affected by the COVID-19 pandemic. Building on and extending these topics, we discuss two areas, which will likely be disrupted by the pandemic for volunteers and their parent organizations: (a) leadership and (b) interactions with community stakeholders and associated challenges for volunteers and volunteering experiences. We conclude with some ideas on how we can tackle 
these challenges using the theories and research on industrial-organizational psychology as applied to the volunteering context.

\section{Challenge 1: Directive Leadership is Good for Pandemic but Bad for Inclusivity}

The COVID-19 pandemic has created unique challenges for leaders managing volunteers. Rudolph et al. (2020) has cited evidence suggesting that directive leadership tends to be particularly effective during times of crises such as financial meltdowns of 2008 (Stoker, Garretsen, \& Soudis, 2019). These predictions are based on threat-rigidity hypothesis, suggesting that organizations respond to external threats such as financial meltdowns or global pandemics with actions that reflect rigidity (Staw, Sandelands, \& Dutton, 1981). In particular, they implement directive leadership, which is based on discouraging participation, tightening control, and centralizing decision-making. Although effective in crises, such a demand-and-control type of leadership is prototypically associated more with men than women (Williams \& Tiedens, 2016) - an association that many volunteering organizations try to break. By contrast, many emergency services that rely on volunteers are aiming to promote an inclusive culture, wherein all volunteers irrespective of their cultural and demographic background, feel supported and valued (Nishii, 2013). Many of these services and leaders therein are striving to attract and retain younger, female, and racially diverse volunteers. Our own research of over 500 emergency services volunteers from Western Australia has supported the beneficial effects of inclusive culture for all volunteers and for women in particular (Gagné et al., 2019). COVID-19 has created a unique challenge in that to effectively manage volunteers during the pandemic leaders must take on directive style. Ironically, such a directive leadership is at risk of damaging a fledgling inclusive culture, which is paramount for attraction and retention of culturally and demographically diverse volunteers. Hence, the current pandemic may slow down the progress on making the volunteering culture more inclusive. 
THE PANDEMIC AND VOLUNTEERS

\section{Challenge 2: Building Community Trust while Complying with Pandemic Rules}

One of the core motivations for volunteers is to give back to the community by helping, educating, and interacting with the community (Kragt, Dunlop, Gagne, Holtrop, \& Luksyte, 2018). Successfully giving back to a community is best achieved by building and maintaining relationships with the community members. However, the social distancing rules may preclude volunteers from building and nourishing such open and trusting relationships with the community. For example, wearing masks and keeping social distancing is a required response to stop the spread of COVID-19. Yet, when volunteers must enter someone's house to fix a roof or deliver goods they may be greeted with suspicion and resistance because their altruistic intentions may be obscured by masks (Tamura, Kishimoto, \& Fukushima, 2013). Further, during the pandemic, volunteers may be called upon to help the police officers to garner compliance with lockdown orders by citizens. To the extent this occurs, trust between the volunteer services and the public may erode, which could decrease volunteers' motivation to serve the community, resulting voluntary exit from volunteering. If such an attrition among volunteers occurs during the pandemic, this adds substantially to the 'everyday' challenges in that volunteer organizations already face with recruiting and retaining volunteers under normal circumstances.

Finally, similar to other disasters, this pandemic has seen a rise in 'spontaneous volunteers' or volunteers who take initiative to help vulnerable members of their communities with transportation and delivering supplies, well-being checks, call centre support, or administration and computing tasks (Butler, 2020). However, while spontaneous volunteers may be driven by altruistic intentions they can cause more harm than help if they do not comply with the social distancing and/ or hygiene rules imposed by the COVID-19 pandemic. By forcing these rules upon spontaneous volunteers, organizations may squash their motivation and future intentions to continue volunteering. 


\section{Solutions to Keep Volunteering Motivation while Complying with the Pandemic}

\section{Restrictions}

Volunteering is a unique experience in that it is mainly driven by intrinsic motivation to be helpful and give back to the community (Kragt \& Holtrop, 2019). As such, it is imperative for volunteer involving organizations to keep their volunteers engaged while also complying with restrictive rules of the COVID-19 pandemic. Research on threat-rigidity hypothesis suggests that directive leadership may be an effective short-term solution, wherein leaders mobilize their efforts to come up with clear guidelines on how to act and behave during a crisis such as the current pandemic. But in the longer run leaders should reinforce their volunteers' motivation to give back to the community, to meaningfully contribute, and be useful. Leaders who use promotion-focused communication during crises, such as focusing on discussing challenges laying ahead, providing hope, and focusing on what can be gained during this pandemic, can sustain their volunteers' motivation while also reinforcing compliance with the pandemic restrictions (Stam, van Knippenberg, Wisse, \& Pieterse, 2018). Such leaders are more effective during crises than those who convey preventionfocused communication, such as emphasizing being vigilant and careful, avoiding errors, and providing safety. One may say that promotion-focused communication may be at odds with directive leadership. But we argue that, once clear rules on how to deal, act, and behave during the pandemic have been established, leaders may further inspire and encourage volunteers by conveying how they can grow as the result of this pandemic.

\section{Satisfying Volunteers' Needs during a Pandemic is Critical}

Another way to keep volunteers' motivation while also ensuring compliance with the pandemic rule can be done via addressing volunteers' needs of (1) autonomy, (2) competence, and (3) relatedness. First, volunteers will continue experiencing sense of autonomy or freedom to perform meaningful tasks if their leaders clearly explain safety rules during a 
pandemic, which directive leadership is good at. Research has shown that nurses are more likely to wash their hands if they are reminded that in doing so they will ensure their patients' safety (Grant \& Hofmann, 2011). Accordingly, directive leaders could also emphasize the importance of following the pandemic rules by highlighting benefits of compliance for not only self but vulnerable community members. Considering the beneficial impact of promotion-focused communication during crises, leaders should share "good news" about the impact of volunteering through personal communications or through social media. Second, to satisfy their competence needs or sense of accomplishment, volunteers could use their newly freed time during a pandemic, wherein, many volunteers' activities are put on hold, for online training to either learn new skills or update their existing ones.

Finally, our own data showed that meaningful social connections and sense of community are one of the central drivers of volunteers' wellbeing and retention (Gagné et al., 2020). Given the centrality of satisfying relatedness needs for volunteers' wellbeing, the imposed social isolation of COVID-19 pandemic may disrupt this goal and may lead to reduced motivation to volunteer. As such, it is critical to keep volunteers being connected to colleagues and to their community by facilitating and encouraging virtual meetings and virtual teamwork with other volunteers and through social media. In doing so, organizations that employ volunteers will ensure that they keep their volunteers engaged, motivated, and connected during the current pandemic as well as develop tools to tackling future crises. 


\section{References}

Butler, P. (2020). A million volunteer to help NHS and others during Covid-19 outbreak. The Guardian. Retrieved from https://www.theguardian.com/society/2020/apr/13/amillion-volunteer-to-help-nhs-and-others-during-covid-19-lockdown

Gagné, M., Luksyte, A., Dunlop, P., Holtrop, D., Kragt, D., \& Muhammad Farid, H. (2019). Emergency volunteer retention: Can a culture of inclusiveness help? Paper presented at the AFAC19 Conference, Melbourne, Australia.

Gagné, M., Luksyte, A., Dunlop, P., Holtrop, D., Muhammad Farid, H., \& Kragt, D. (2020). The influence of state emergency volunteers' age on their psychological needs, wellbeing and retention. Paper presented at the Academy of Management Annual Meeting

Grant, A. M., \& Hofmann, D. A. (2011). It's not all about me: Motivating hand hygiene among health care professionals by focusing on patients. Psychological Science, 22, 1494-1499. doi:10.1177/0956797611419172

Kragt, D., Dunlop, P., Gagne, M., Holtrop, D., \& Luksyte, A. (2018). When joining is not enough: emergency services volunteers and the intention to remain. Australian Journal of Emergency Management, 33, 35-40.

Kragt, D., \& Holtrop, D. (2019). Volunteering research in Australia: A narrative review. Australian Journal of Psychology, 71, 342-360. doi:10.1111/ajpy.12251

Nishii, L. H. (2013). The benefits of climate for inclusion for gender-diverse groups. Academy of Management Journal, 56, 1754-1774. doi:10.5465/amj.2009.0823

Services, D. o. F. a. E. (2019). Annual Report 2018/19. Retrieved from https://www.dfes.wa.gov.au/publications/Annual\%20Reports/DFES_Annual_Report2018-19.pdf 
Stam, D., van Knippenberg, D., Wisse, B., \& Pieterse, A. N. (2018). Motivation in words: Promotion- and prevention-oriented leader communication in times of crisis. Journal of Management, 44, 2859-2887. doi:10.1177/0149206316654543

Staw, B. M., Sandelands, L. E., \& Dutton, J. E. (1981). Threat-rigidity effects in organizational-behavior - A multilevel analysis. Administrative Science Quarterly, 26, 501-524. doi:10.2307/2392337

Stoker, J. I., Garretsen, H., \& Soudis, D. (2019). Tightening the leash after a threat: A multilevel event study on leadership behavior following the financial crisis. Leadership Quarterly, 30, 199-214. doi:10.1016/j.leaqua.2018.08.004

Tamura, E., Kishimoto, K., \& Fukushima, N. (2013). Effect of pharmacists wearing a mask on patient consultation intention. Journal of the Pharmaceutical Society of Japan, 133, 737-745. doi:10.1248/yakushi.12-00271

Volunteers, U. N. (2018). State of the World's Volunteerism Report 2018: The Thread that Binds. Retrieved from https://www.unv.org/sites/default/files/UNV_SWVR_2018_English_WEB.pdf

Williams, M. J., \& Tiedens, L. Z. (2016). The subtle suspension of backlash: A meta-analysis of penalties for women's implicit and explicit dominance behavior. Psychological Bulletin, 142, 165-197. doi:10.1037/bul0000039

Wilson, J. (2000). Volunteering. Annual Review of Sociology, 26, 215-240. doi:0.1146/annurev.soc.26.1.215 